\title{
The crosstalk of ABCA1 and ANXA1: a potential mechanism for protection against atherosclerosis
}

\author{
Xin Shen ${ }^{1+}$, Shun Zhang ${ }^{1 \dagger}$, Zhu Guo ${ }^{1,2}$, Dongming Xing ${ }^{1,3^{*}}$ and Wujun Chen ${ }^{1 *}$ (D)
}

\begin{abstract}
Atherosclerosis, characterized by the formation of fat-laden plaques, is a chronic inflammatory disease. ABCA1 promotes cholesterol efflux, reduces cellular cholesterol accumulation, and regulates anti-inflammatory activities in an apoA-I- or ANXA1-dependent manner. The latter activity occurs by mediating the efflux of ANXA1, which plays a critical role in anti-inflammatory effects, cholesterol transport, exosome and microparticle secretion, and apoptotic cell clearance. ApoA-I increases ANXA1 expression via the ERK, P38MAPK, AKT, and PKC pathways. ApoA-I regulates the signaling pathways by binding to $A B C A 1$, suggesting that apoA-I increases ANXA1 expression by binding to ABCA1. Furthermore, ANXA1 may increase ABCA1 expression. ANXA1 increases PPARy expression by modulating STAT6 phosphorylation. PPARY also increases ANXA1 expression by binding to the promoter of ANXA1. Therefore, ABCA1, PPAR $\gamma$, and ANXA1 may form a feedback loop and regulate each other. Interestingly, the ANXA1 needs to be externalized to the cell membrane or secreted into the extracellular fluids to exert its anti-inflammatory properties. ABCA1 transports ANXA1 from the cytoplasm to the cell membrane by regulating lipidization and serine phosphorylation, thereby mediating ANXA1 efflux, likely by promoting microparticle and exosome release. The direct role of ABCA1 expression and ANXA1 release in atherosclerosis has been unclear. In this review, we focus on the role of ANXA1 in atheroprogression and its novel interaction with ABCA1, which may be useful for providing basic knowledge for the development of novel therapeutic targets for atherosclerosis and cardiovascular disease.
\end{abstract}

Keywords: ANXA1, ABCA1, PPARY, Crosstalk, Atherosclerosis

\section{Introduction}

Cardiovascular diseases (CVDs), including coronary artery disease (CAD), myocardial infarction $(\mathrm{MI})$, and heart failure (HF), are the leading cause of death in humans and are manly caused by atherosclerosis. Atherosclerosis, characterized by the formation of fat-laden plaques in large- and medium-sized vessels, has been identified as a chronic inflammatory disease of the artery wall (Saigusa et al. 2020). Atherosclerosis occurs through the recruitment of monocytes and their differentiation into macrophages, which require lipids for foam cell

\footnotetext{
*Correspondence: xdm_tsinghua@163.com; cwjwxt5951@163.com

${ }^{+}$Xin Shen and Shun Zhang contributed equally to this work.

${ }^{1}$ Cancer Institute, The Affiliated Hospital of Qingdao University, Qingdao University, Qingdao Cancer Institute, Qingdao 266071, Shandong, China Full list of author information is available at the end of the article
}

generation, a process regulated by balancing the rates of lipid uptake and efflux (Peng et al. 2020). The latter is mainly controlled by ATP binding cassette transporter A1 (ABCA1), a membrane transporter that is abundant in macrophages and mediates cholesterol efflux (Wang et al. 2020). ABCA1 promotes the efflux of free cholesterol to apolipoprotein A-I (apoA-I) and is critical for high-density lipoprotein (HDL) particle biogenesis. These particles protect against atherosclerotic vascular diseases by transferring cholesterol from peripheral cells to the liver for biliary excretion, constituting the process of reverse cholesterol transport (Libby et al. 2019; Ahmadi et al. 2019).

Recently, several studies have shown that ABCA1 reduces inflammatory responses by inhibiting the secretion

(c) The Author(s). 2020 Open Access This article is licensed under a Creative Commons Attribution 4.0 International License, which permits use, sharing, adaptation, distribution and reproduction in any medium or format, as long as you give

appropriate credit to the original author(s) and the source, provide a link to the Creative Commons licence, and indicate if changes were made. The images or other third party material in this article are included in the article's Creative Commons licence, unless indicated otherwise in a credit line to the material. If material is not included in the article's Creative Commons licence and your intended use is not permitted by statutory regulation or exceeds the permitted use, you will need to obtain permission directly from the copyright holder. To view a copy of this licence, visit http://creativecommons.org/licenses/by/4.0/. 
of interleukin-1 $\beta$ (IL-1 $\beta$ ) and tumor necrosis factor (TNF- $\alpha$ ) and the NF- $\mathrm{kB}$ signaling pathway via the removal of reactive oxygen species (ROS) (Babashamsi et al. 2019), and ABCA1 may be linked to these functions via apoA-I. Interestingly, ABCA1, in addition to mediating cholesterol efflux, promotes the secretion of annexin $A 1$ (ANXA1), which is associated with antiinflammatory reactions, cell differentiation and proliferation, cell phagocytosis and clearance, cholesterol homeostasis, immunogenic cell death (ICD), autophagy, apoptosis, and exosomes (Chapman et al. 2003; Omer et al. 2006; Li et al. 2018). ANXA1 is also a specific marker of microparticles, and accumulating evidence has shown that ANXA1 plays a key role in inhibiting the development of atherosclerosis via an anti-inflammatory reaction (Jeppesen et al. 2019). The ANXA1 mimetics Ac2-26 and CGEN-855A have also been shown to protect against atherosclerosis and $\mathrm{MI}$ progression via an anti-inflammatory reaction. CGEN-855A (TIPMFVPEST SKLQKFTSWFM-amide) is a novel 21-amino acid mimetic peptide of ANXA1. CGEN-855A was developed by Compugen Ltd., Tel Aviv, Israel (Hecht et al. 2009; Qin et al. 2015). The anti-inflammatory and cardio protective effects of CGEN-855A have been investigated by pharmaceutical companies, but the direct effects of ANXA1 on cholesterol transport, exosome and microparticle secretion, apoptotic cell clearance, and ICD in atherosclerosis have rarely been investigated. Although ABCA1 mediates ANXA1 release, the effect of this function on atherosclerosis has been unclear. In addition, ANXA1 may increase ABCA1 expression, but the effect on atherosclerosis development is unknown (Zhou et al. 2009; da Rocha et al. 2019; Chawla et al. 2001; Parente and Solito 2004). In this article, we review the roles of ANXA1 in atherosclerosis and focus on the crosstalk of ABCA1 and ANXA1. We expect to promote the study of the role of ANXA1 in atherosclerosis and sincerely hope that more scientists will study the relationship of ABCA1 with ANXA1 and atherosclerosis development.

\section{ANXA1 mediates anti-inflammatory effects, cholesterol transport, exosome and microparticle secretion, and apoptotic cell clearance}

ANXA1, the first identified member of the annexin family, is an anti-phospholipase protein that has $\mathrm{Ca}^{2+}$ and phospholipid binding sites and high biological and structural homology to other family members (Parente and Solito 2004). ANXA1 is highly expressed in neutrophils and monocytes/macrophages, and its anti-inflammatory activity is associated with interference of the leukocyte migration and platelet aggregation (Parente and Solito 2004; Senchenkova et al. 2019). ANXA1 was predominantly found within gelatinase granules and in the cytoplasm (Perretti et al. 2000; Solito et al. 2006). ANXA1 binds to and activates the formyl peptide receptor (FPR) on leukocytes and endothelial cells, thereby inhibiting leukocyte migration to inflammatory sites (Senchenkova et al. 2019; Parisi et al. 2019; Pessolano et al. 2019). ANXA1 also reduces inflammatory responses by inhibiting phospholipase $\mathrm{A}_{2}\left(\mathrm{PLA}_{2}\right)$, thereby inhibiting the synthesis of arachidonic acid (AA) and AA-derived metabolites, including thromboxane $\mathrm{A} 2\left(\mathrm{TXA}_{2}\right)$, prostaglandin $\mathrm{E}_{2}\left(\mathrm{PGE}_{2}\right), \mathrm{PGI}_{2}, \mathrm{PGD}_{2}$, and PGF2 $\alpha$ (Sanches et al. 2020; Seidel et al. 2012). ANXA1 also prevents inflammatory responses by reducing nitric oxide synthase (iNOS) and the expression of cyclooxygenase-2 (COX2 ), which is a rate-limiting key enzyme of prostaglandin synthesis (Seidel et al. 2012; Kiani-Esfahani et al. 2016). ANXA1 also induces macrophage polarization toward a more anti-inflammatory phenotype that secretes IL-10, an anti-inflammatory cytokine (Ferlazzo et al. 2003; Butcher and Galkina 2015; Giannarelli and Wong 2019). Thus, ANXA1 functions as an anti-inflammatory protein via multiple mechanisms, and many studies suggest that ANXA1 reduces atherosclerosis progression via an antiinflammatory reaction. In human coronary atherosclerotic plaques, ANXA1 was found to localize in macrophages and endothelial cells in the tunica intima (de Jong et al. 2017a). The expression of the ANXA1 gene in the carotid plaques of asymptomatic patients was significantly higher than in symptomatic patients (Cheuk and Cheng 2011). The actions of ANXA1, which are mediated through its receptor FPR2, can be mimicked by an amino-terminal peptide encompassing Ac2-26, demonstrating a protective effect of ANXA1 on the early stage of plaque development (Kusters et al. 2015; de Jong et al. 2017b).

Recently, apoA-I was found to increase ANXA1 expression via the ERK, p38 MAPK, AKT, and PKC pathways in human umbilical vein endothelial cells (HUVECs) (Pan et al. 2016). ApoA-I can bind to ABCA1 and then influence several signaling pathways, including ERK, p38 MAPK, AKT, and PKC, and the synthesis of apoA-I protein is generally thought to occur in the liver and small intestine. However, apoA-I is also transcribed in macrophages, and the endogenous apoA-I in macrophages has an anti-inflammatory effect, suggesting that ABCA1 increases ANXA1 expression through binding to apoA-I, controlling the ERK, p38 MAPK, AKT, and PKC pathways (Chen et al. 2020). The drugs associated with apoA-I that are used to treat CVD, particularly CAD, have an apoA-I mimetic peptide, including D-4F, L-4F, 6-F, 18A, 37pA, 5A ETC-642, FAMP, apoA-I milano including MDCO-216 (Phase 2, Termination), rHDL particles that comprise human plasma-derived apoA-I and soybean phosphatidylcholine including CSL111 (Phase 2) and CSL112 (Phase 3), HDL mimetics that comprise recombinant full-length human apoA-I and two natural 
phospholipids including CER-001 (Phase 2), and BETinhibitor including RVX-208, which significantly stimulate apoA-I transcription (Phase 3) (Yu et al. 2019). Many studies have shown that these drugs have an antiinflammatory effect by increasing apoA-I expression (Yu et al. 2019). Based on the observed strong activation of apoA-I with ANXA1 expression, these drugs may augment the anti-inflammatory effect by increasing the apoA-I/ANXA1 axis. Therefore, we hypothesize that the drugs associated with apoA-I have multiple functions stimulating the apoA-I/ANXA1 axis, including antiinflammatory effects, cholesterol transport, exosome and microparticle secretion, apoptotic cell clearance, and ICD. According to this hypothesis, new drug targets may exist, but more research is needed.

Many studies have shown a relationship between ANXA1 and lipid metabolism. ANXA1 expression is strongly increased in adipose tissue, including in mice fed a high-fat diet (Akasheh et al. 2013) and obese children (Aguilera et al. 2015), and in the subcutaneous fat of young and old overweight patients (Alfadda et al. 2013). ANXA1 expression is also increased in the livers of mice with nonalcoholic steatohepatitis (NASH), which is characterized by hepatic lipid accumulation (Locatelli et al. 2014). The plasma levels of ANXA1 in patients with type1 diabetes (T1D) and T2D are significantly higher than in control patients. In addition, the levels of ANXA1 in T2D patients are positively correlated with serum low-density lipoprotein cholesterol (LDL-C) levels, total cholesterol levels, and the fatty liver index (FLI). ANXA1-/- mice have significantly higher serum triglyceride levels, more extensive Oil Red-O staining in the liver, and are more obese than wild-type mice, and administration of hrANXA1 to these mice reduces serum cholesterol and liver triglyceride levels, the extent of Oil Red-O staining in liver, and weight gain (Purvis et al. 2019a). These findings may suggest that ANXA1 is associated with lipid and glucose metabolism. Moreover, ANXA1 mediates cholesterol transport from the endoplasmic reticulum (ER) to multivesicular bodies (MVB) and then stimulates the secretion of exosomes from MVBs via endosomal-sorting complexes required for transport (ESCRT) and Alg-2 interacting protein $\mathrm{X}$ (Alix) (Eden et al. 2016; Rentero et al. 2018). Interestingly, exosomes also carry ANXA1 (Rentero et al. 2018; Raulf et al. 2018). Exosomes protect and transport lipids, proteins, and RNAs, fostering intercellular communication among different cell types involved in atherosclerosis, such as macrophages, endothelial cells, and smooth muscle cells. Many studies suggest that exosomes could be used for not only the diagnosis but also the treatment of atherosclerosis (Reiss et al. 2017; Wang et al. 2019), but whether the interaction of ANXA1 with exosomes also regulates atherosclerosis progression needs to be further studied.

ANXA1 was also found in microparticles (also known as microvesicles) from complex vesicular structures shed from endothelial cells, insulin-secreting Rin-m $5 f \beta$-cells, ATRA-NB4 cells, neutrophils, and leukocytes (Kreutter et al. 2017; Nadkarni et al. 2011; Tsai et al. 2012; Tsai et al. 2013), and it serves as a specific marker of microparticles shed from the plasma membrane (Jeppesen et al. 2019). However, the role of ANXA1 in microparticles has not been investigated. Microparticles, heterogeneous extracellular vesicles that include exosomes, nanoparticles, and shedding vesicles, are not only a prognostic marker of atherosclerosis acceleration and clinical presentation of familial hypercholesterolemia but also therapeutic agents for CAD (Boulanger et al. 2017; Loyer et al. 2014; Suades et al. 2019). Therefore, ANXA1 could regulate atherosclerosis development by mediating the transport of cholesterol and the secretion of exosomes and microparticles. However, the relationship between ANXA1-mediated cholesterol transport and the secretion of exosomes and microparticles is unclear.

ANXA1 is also an ICD hallmark. The ANXA1 secreted from apoptotic cells binds to the FRP1 receptor on antigen-presenting cells (APCs) and then helps guide the APCs to the dying cells to promote their clearance (Cruickshank et al. 2018). Many studies have demonstrated that impaired clearance of apoptotic cells promotes the development of atherosclerosis, suggesting that the ICD process by ANXA1 may play a key role in atherosclerosis development (Aprahamian et al. 2004; Poon et al. 2014). Intensive studies of ICD have focused on cancer, leaving its role in atherosclerosis development largely unelucidated.

\section{The crosstalk between ABCA1 expression and ANXA1 efflux}

ANXA1 secretion has been found to occur in many types of cells, including monocytes/macrophages, mast cells, epithelial cells, folliculo-stellate (FS) cells, neutrophils, and astrocytes (Purvis et al. 2019b; Sugimoto et al. 2016). It has also been detected in a variety of tissues, such as heart, artery, skeletal muscle, small intestine, colon, adipose, kidney, liver, lung, spleen, stomach, pancreas, skin, brain, and prostate tissue, wherein ABCA1 is strongly expressed (Frambach et al. 2020). In addition, the expression of ABCA1 and ANXA1 are positively correlated in plaque macrophages with oxidized low-density lipoprotein (oxLDL) and monocytes of hypercholesterolemic pigs (Geeraert et al. 2007; Orekhov et al. 2018). Traditionally, ABCA1 has been mainly found to promote cholesterol efflux, and its anti-inflammatory effect is associated with apoA-I (Phillips 2018). Previous studies from our laboratory and others have demonstrated that 
the ABCA1 complex with apoA-I plays a critical role in cholesterol efflux and anti-inflammatory activities (Kuang et al. 2017; Yin et al. 2012; Chen et al. 2013; Tang et al. 2012; Price et al. 2019). Interestingly, ANXA1 secretion has been shown to require ABCA1 in macrophages and endocrine cells. The ABCA1 inhibitor glyburide blocks ANXA1 secretion (Seidel et al. 2012). Chapman et al. revealed that ABCA1 promotes ANXA1 release in mouse FS and A549 cells. ANXA1 interacts with ABCA1 via ANXA1 amino acids 196 to 274 and colocalizes with ABCA1 in the cell plasma membrane (Chapman et al. 2003), and an ABCA1 inhibitor and siRNA were shown to significantly reduce the membrane localization of ANXA1. In contrast, ABCA1 overexpression in AtT20 D1 FS cells significantly increases plasma membrane-associated ANXA1 expression (Omer et al. 2006). Importantly, ABCA1 mediated the secretion of ANXA1 in Hensen cells, in which the majority of ANXA1 is stored inside lipid droplets. Myosin II promotes ANXA1 translation from lipid droplets to the apical region of Hensen cells, in which ABCA1 facilitates the release of ANXA1 to the external milieu (Kalinec et al. 2009). Of note, ischemia-reperfusion injury increases ABCA1 ubiquitination and degradation in the ganglion cell layer, leading to decreased ANXA1 translocation to the cell membrane, and its secretion facilitates retinal inflammation and retinal ganglion cell apoptosis (Xiao et al. 2018). Notably, the LXR agonist T0901317 induces ICD by increasing calreticulin (CRT) and high mobility group protein (HMGB1) in colon cancer cells (Wang et al. 2018a). ABCA1 is a target gene of LXR. T0901317 also increases ABCA1 expression. ABCA1 promotes ANXA1 release, suggesting that the ABCA1/ANXA1 axis plays a critical role in the ICD by T0901317. Taken together, these data show that ABCA1 promotes the efflux of ANXA1, which is critical for the activities of ANXA1.

Importantly, the ANXA1 needs to be externalized to the cell membrane or secreted into the extracellular fluids to exert its anti-inflammatory properties. The transport of ANXA1 from the cytoplasm to the cell membrane is dependent on serine phosphorylation and lipidization (Solito et al. 2006). ABCA1 is a key regulator of apoA-I (Wang et al. 2013) and apoE lipidation (Krimbou et al. 2004), and ABCA1 also contains serine residues in its intracellular segment; the phosphorylation of ABCA1 serine residues can increase cholesterol efflux (Hu et al. 2009). ABCA1 mediates the secretion of ANXA1, which is stored in lipid droplets and localized in microparticles and exosomes. ABCA1 also mediates microparticle (Duong et al. 2006; Nandi et al. 2009; Hafiane and Genest 2017) and exosome secretion (Hafiane and Genest 2017; Ma et al. 2011). ABCA1 transports ANXA1 from the cytoplasm to the cell membrane, likely by regulating lipidization and serine phosphorylation, thereby mediating ANXA1 efflux most likely by promoting microparticle and exosome release. However, the role of the colocalization of both proteins on the plasma membranes during ANXA1 efflux needs to be determined. This hypothesis may be correct, at least $\mathrm{ABCA} 1$ transports ANXA1 from the cytoplasm to the cell membrane. Thus, ABCA1-mediated ANXA1 transport and release play a key role in the antiinflammatory action of ANXA1.

Interestingly, enhanced AA production through upregulation of cPLA 2 activity has been shown to inhibit LXR expression and thereby reduce $\mathrm{ABCA} 1$ expression and cholesterol efflux (Zhou et al. 2009). As mentioned before, ANXA1, which serves as the inhibitory protein of PLA2, can reduce the synthesis of AA. ANXA1-/- mice have shown increased serum triglyceride levels and Oil Red-O staining of the liver as well as enhanced obesity compared with wild-type mice. Administration of hrANXA1 to mice reduced serum cholesterol and liver triglyceride levels, Oil Red-O staining in the liver, and weight gain, suggesting that ANXA1 increases ABCA1 expression and cholesterol efflux. In addition, peroxisome proliferator-activated receptor-gamma (PPAR $\gamma$ ), which has been implicated in lipid metabolism, the inflammatory response, and glucose homeostasis, is increased in ANXA1-/- mice, suggesting a role for ANXA1 in mediating PPAR $\gamma$ expression (Akasheh et al. 2013). ANXA1 increases PPARY and CD36 expression by modulating STAT6 phosphorylation in BV2 cells, subsequently mediating phagocytosis of apoptotic cells (da Rocha et al. 2019). Macrophage CD36 participates in atherosclerotic arterial lesion formation via the promotion of oxLDL uptake and foam cell formation. However, ABCA1 and CD36 are induced by PPARy activation. Thus, PPAR $\gamma$ controls intracellular cholesterol balance, including cholesterol uptake, processing, and cholesterol removal, through $\mathrm{ABCA} 1$ and $\mathrm{CD} 36$ (Chawla et al. 2001). IL-10 also increases CD36 and ABCA1 expression, thereby facilitating cholesterol uptake (minor) and efflux (major) (Han et al. 2009). IL-19, which has a similar exon-intron structure and shares a $21 \%$ amino acid identity with IL-10, also increases ABCA1 and ABCG1 expression and cholesterol efflux (Gabunia et al. 2016). Administration of $10 \mathrm{ng} / \mathrm{g} /$ day IL-19 to LDLR-/- mice almost completely inhibits plaque formation in the aortic arch, and IL-19 at as little as $1 \mathrm{ng} / \mathrm{g} /$ day reduces the plaque area by $70 \%$, suggesting that IL-19 has a strong anti-atherosclerotic effect (Ellison et al. 2013). Mechanistic studies have shown that IL-19 reduces cytokineinduced inflammation by promoting the activation of STAT3, STAT6, Kruppel-like factor 4 (KLF4), and PPAR $\gamma$ (Gabunia et al. 2016). IL-19 regulates lipid metabolism via the PPAR $\gamma$-dependent regulation of CD36- 
mediated cholesterol uptake and ABCA1-mediated cholesterol efflux (Gabunia et al. 2016). IL-19 reduces oxLDL uptake by inducing the expression of miR-133a to reduce the expression of the target gene low-density lipoprotein receptor adaptor protein 1 (LDLRAP1), which functions to internalize the LDL receptor (Ellison et al. 2013; Gabunia et al. 2017). IL-19 reduces TNF $\alpha$ expression by inducing the expression of miR-133a to reduce the expression of human antigen $\mathrm{R}(\mathrm{HuR})$, which is an important regulator of TNF $\alpha$ mRNA stability (Ray et al. 2018), suggesting that IL-19 is a link between inflammation and cholesterol metabolism. In addition, ANXA1 decreases the expression levels of some miRNAs in MCF-7 cells, including miR-26b*, miR-27a, miR-200c, and miR-562 (Anbalagan et al. 2014). Previous studies by our laboratory and others have demonstrated that miRNAs, including miR-27a and miR-10b, decrease cholesterol efflux by binding to the 3'UTR of ABCA1 (Zhang et al. 2014; Wang et al. 2018b; Wu et al. 2020). Based on the aforementioned information, we hypothesize that ANXA1 can also increase ABCA1 expression by regulating the expression of PPAR $\gamma, \mathrm{IL}-10$, and miRNAs, and then regulate cholesterol uptake and efflux. Given that ANXA1 is a protective factor for atherosclerosis, its role in cholesterol efflux may be dominant. Interestingly, PPAR $\gamma$ has also been shown to increase ANXA1 expression by binding to the promoter of ANXA1 in breast cancer cells (Chen et al. 2017). Thus, ABCA1, PPAR $\gamma$, and ANXA1 may form a feedback loop and regulate each other. Notably, PPARy

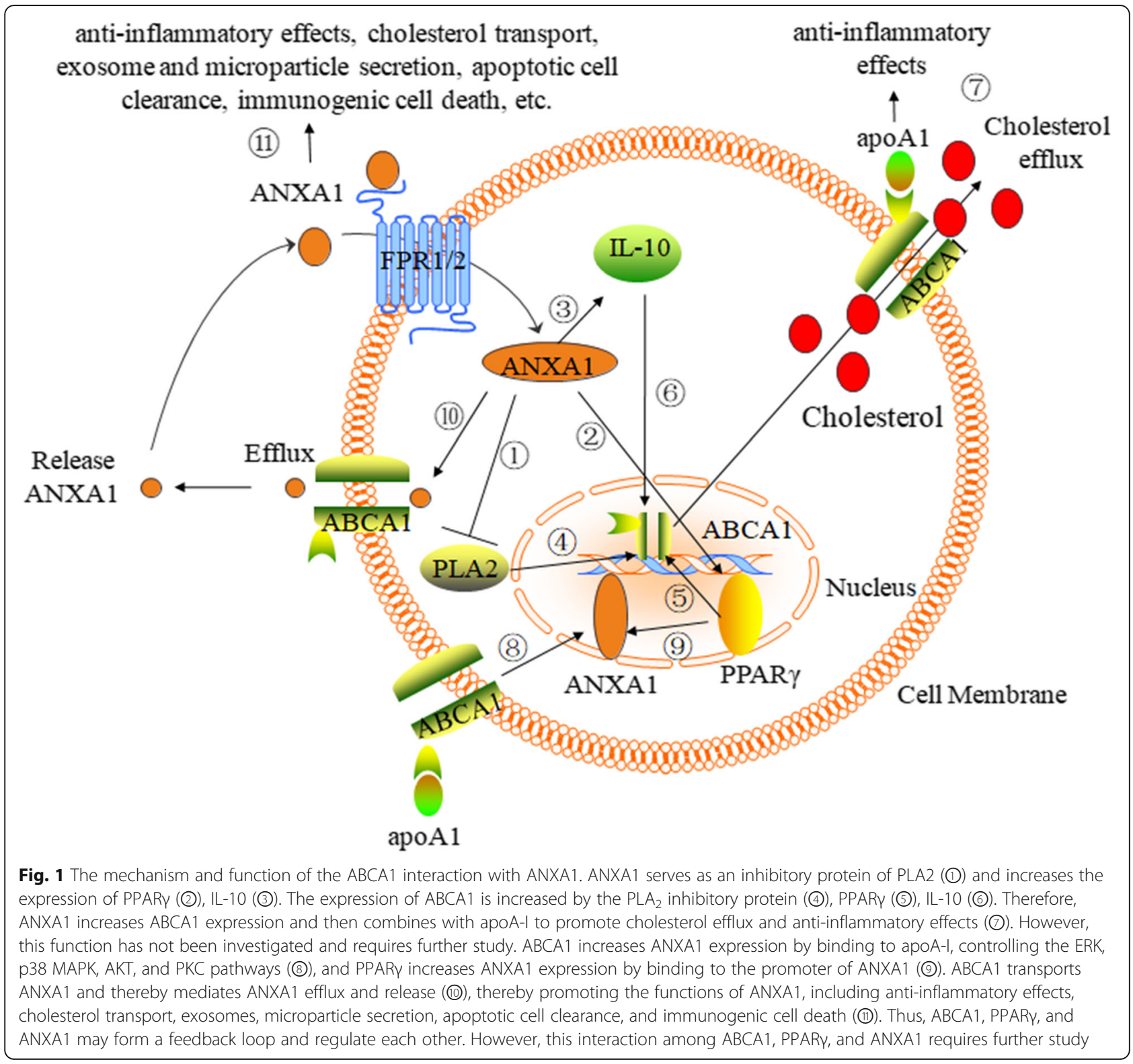


induces cholesterol efflux by binding to LXRa and controlling the expression of the target gene ABCA1 (Wang et al. 2018c; Tsuboi et al. 2020), suggesting that PPAR $\gamma$ may play a primary role in the feedback loop. However, this interaction among ABCA1, PPAR $\gamma$, and ANXA1 requires further study.

\section{Summary}

ANXA1-mediated anti-inflammatory effects, cholesterol transport, exosome and microparticle secretion, and apoptotic cell clearance play key roles in atheroprogression. Importantly, $\mathrm{ABCA} 1$, in addition to mediating cholesterol efflux and anti-inflammatory effects by binding to apoA-I, increases ANXA1 expression and transports ANXA1 from the cytoplasm to the cell membrane, thereby promoting ANXA1 into the extracellular fluids to facilitate anti-inflammatory activities and apoptotic cell clearance. ANXA1 may also increase ABCA1 expression and cholesterol efflux by serving as the $\mathrm{PLA}_{2}$ inhibitory protein or increasing PPAR $\gamma$ and IL-10 expression. ABCA1, PPAR $\gamma$, and ANXA1 may form a feedback loop and regulate each other (Fig. 1). Despite this evidence for the relationship of mutual regulation between ABCA1 expression and ANXA1 efflux, less is known regarding the potential role of this mutual regulatory effect in cardiovascular disease. Further studies need to focus on the role of ABCA1-mediated ANXA1 efflux and ANXA1-mediated ABCA1 expression in the development of atherosclerosis. In addition, ABCA1 is not the only transporter that mediates ANXA1 secretion, as $\mathrm{ABCC} 1$ also mediates this phenomenon (Wein et al. 2004). The relationship between ABCA1 and ABCC1 during ANXA1 efflux requires further study. Taken together, these observations suggest that crosstalk may exist between ANXA1 and ABCA1, IL-10, and PPAR $\gamma$. However, the direct association of ANXA1 with increased ABCA1 expression has not been investigated. Finally, we sincerely hope that many more scientists will focus on crosstalk in atherosclerosis.

\footnotetext{
Abbreviations

CVD: Cardiovascular disease; CAD: Coronary artery disease; MI: Myocardial infarction; HF: Heart failure; ABCA1: ATP binding cassette transporter A1; ApoA-I: Apolipoprotein A-l; HDL: High-density lipoprotein; IL-1: Interleukin-1; TNF-a: Tumor necrosis factor; ROS: Reactive oxygen species; ANXA1: Annexin A1; ICD: Immunogenic cell death; FPR: Formyl peptide receptor; PLA $A_{2}$ : Phospholipase $A_{2} ; A A$ : Arachidonic acid; TXA $\mathrm{PGE}_{2}$ : Prostaglandin $\mathrm{E}_{2}$; iNOS: Nitric oxide synthase; COX-2: Cyclooxygenase-2; ER: Endoplasmic reticulum; MVB: Multivesicular bodies; ESCRT: Endosomalsorting complexes required for transport; APCs: Antigen-presenting cells; oxLDL: Oxidized low-density lipoprotein; FS: Folliculo-stellate;

PPARY: Peroxisome proliferator-activated receptor-gamma
}

\section{Acknowledgments}

We thank the laboratory of Professor Dongming Xing for generously supplying reagents, technical assistance, stimulating discussions, and funding.

\section{Authors' contributions}

Conception and design: Dongming Xing and Wujun Chen. Manuscript writing: Xin Shen and Shun Zhang. Collection and assembly of data: Zhu Guo. Final approval of the manuscript: All authors.

\section{Funding}

The authors are grateful for the financial support provided by the Qingdao Major Scientific and Technological Project for Distinguished Scholars (20170103), the Laoshan Major Scientific and Technological Project for Distinguished Scholars (20181030), Youth Innovation Team Talent Introduction Program of Shandong Province (20190164), the China Postdoctoral Science Foundation (2019 M652331, 2018 M642619), the Qingdao Postdoctoral Application Project (RZ1900013298, 2018121236, 2018121238).

\section{Availability of data and materials \\ Not applicable.}

\section{Ethics approval and consent to participate}

Not applicable.

\section{Consent for publication}

No individual personal data are involved in this review.

\section{Author details}

${ }^{1}$ Cancer Institute, The Affiliated Hospital of Qingdao University, Qingdao University, Qingdao Cancer Institute, Qingdao 266071, Shandong, China. ${ }^{2}$ Department of Spine Surgery, The Affiliated Hospital of Qingdao University, Qingdao 266071, Shandong, China. ${ }^{3}$ School of Life Sciences, Tsinghua University, Beijing 100084, China.

Received: 12 March 2020 Accepted: 26 August 2020

Published online: 07 September 2020

\section{References}

Aguilera CM, Gomez-Llorente C, Tofe I, Gil-Campos M, Canete R, Gil A. Genomewide expression in visceral adipose tissue from obese prepubertal children. Int J Mol Sci. 2015;16:7723-37.

Ahmadi A, Argulian E, Leipsic J, Newby DE, Narula J. From subclinical atherosclerosis to plaque progression and acute coronary events: JACC stateof-the-art review. J Am Coll Cardiol. 2019;74:1608-17.

Akasheh RT, Pini M, Pang J, Fantuzzi G. Increased adiposity in annexin A1deficient mice. PLoS One. 2013;8:e82608.

Alfadda AA, Benabdelkamel H, Masood A, et al. Proteomic analysis of mature adipocytes from obese patients in relation to aging. Exp Gerontol. 2013;48: 1196-203.

Anbalagan D, Yap G, Yuan Y, et al. Annexin-A1 regulates microRNA-26b* and microRNA-562 to directly target NF-kappaB and angiogenesis in breast cancer cells. PLoS One. 2014;9:e114507.

Aprahamian T, Rifkin I, Bonegio R, et al. Impaired clearance of apoptotic cells promotes synergy between atherogenesis and autoimmune disease. J Exp Med. 2004;199:1121-31.

Babashamsi MM, Koukhaloo SZ, Halalkhor S, Salimi A, Babashamsi M. ABCA1 and metabolic syndrome; a review of the ABCA1 role in HDL-VLDL production, insulin-glucose homeostasis, inflammation and obesity. Diabetes Metab Syndr. 2019;13:1529-34.

Boulanger CM, Loyer X, Rautou PE, Amabile N. Extracellular vesicles in coronary artery disease. Nat Rev Cardiol. 2017;14:259-72.

Butcher MJ, Galkina EV. wRAPping up early monocyte and neutrophil recruitment in atherogenesis via Annexin A1/FPR2 signaling. Circ Res. 2015;116:774-7.

Chapman LP, Epton MJ, Buckingham JC, Morris JF, Christian HC. Evidence for a role of the adenosine $5^{\prime}$-triphosphate-binding cassette transporter $\mathrm{A} 1$ in the externalization of annexin I from pituitary folliculo-stellate cells. Endocrinology. 2003;144:1062-73.

Chawla A, Boisvert WA, Lee CH, et al. A PPAR gamma-LXR-ABCA1 pathway in macrophages is involved in cholesterol efflux and atherogenesis. Mol Cell. 2001;7:161-71.

Chen L, Yuan Y, Kar S, et al. PPARgamma ligand-induced Annexin A1 expression determines chemotherapy response via Deubiquitination of death domain kinase RIP in triple-negative breast cancers. Mol Cancer Ther. 2017;16:2528-42. 
Chen W, Wu Y, Lu Q, Wang S, Xing D. Endogenous ApoA-I expression in macrophages: a potential target for protection against atherosclerosis. Clin Chim Acta. 2020;505:55-9.

Chen WJ, Zhang M, Zhao GJ, et al. MicroRNA-33 in atherosclerosis etiology and pathophysiology. Atherosclerosis. 2013;227:201-8.

Cheuk BL, Cheng SW. Annexin A1 expression in atherosclerotic carotid plaques and its relationship with plaque characteristics. Eur J Vasc Endovasc Surg. 2011:41:364-71.

Cruickshank B, Giacomantonio M, Marcato P, McFarland S, Pol J, Gujar S. Dying to be noticed: epigenetic regulation of immunogenic cell death for cancer immunotherapy. Front Immunol. 2018;9:654.

da Rocha GHO, Loiola RA, Pantaleao LDN, Reutelingsperger C, Solito E, Farsky SHP. Control of expression and activity of peroxisome proliferatedactivated receptor gamma by Annexin A1 on microglia during efferocytosis. Cell Biochem Funct. 2019;37:560-8.

de Jong R, Leoni G, Drechsler M, Soehnlein O. The advantageous role of annexin A1 in cardiovascular disease. Cell Adh Migr. 2017a;11:261-74.

de Jong RJ, Paulin N, Lemnitzer $P$, et al. Protective aptitude of Annexin A1 in arterial Neointima formation in atherosclerosis-prone mice-brief report. Arterioscler Thromb Vasc Biol. 2017b;37:312-5.

Duong PT, Collins HL, Nickel M, Lund-Katz S, Rothblat GH, Phillips MC. Characterization of nascent $\mathrm{HDL}$ particles and microparticles formed by ABCA1mediated efflux of cellular lipids to apoA-I. J Lipid Res. 2006;47:832-43.

Eden ER, Sanchez-Heras E, Tsapara A, Sobota A, Levine TP, Futter CE. Annexin A1 tethers membrane contact sites that mediate ER to endosome cholesterol transport. Dev Cell. 2016;37:473-83.

Ellison S, Gabunia K, Kelemen SE, et al. Attenuation of experimental atherosclerosis by interleukin-19. Arterioscler Thromb Vasc Biol. 2013;33:2316-24.

Ferlazzo V, D'Agostino P, Milano S, et al. Anti-inflammatory effects of annexin-1: stimulation of IL-10 release and inhibition of nitric oxide synthesis. Int Immunopharmacol. 2003;3:1363-9.

Frambach S, de Haas R, Smeitink JAM, Rongen GA, Russel FGM, Schirris TJJ. Brothers in arms: ABCA1- and ABCG1-mediated cholesterol efflux as promising targets in cardiovascular disease treatment. Pharmacol Rev. 2020; 72:152-90.

Gabunia K, Ellison S, Kelemen S, et al. IL-19 halts progression of atherosclerotic plaque, polarizes, and increases cholesterol uptake and efflux in macrophages. Am J Pathol. 2016;186:1361-74.

Gabunia K, Herman AB, Ray M, et al. Induction of MiR133a expression by IL-19 targets LDLRAP1 and reduces oxLDL uptake in VSMC. J Mol Cell Cardiol. 2017; 105:38-48

Geeraert B, De Keyzer D, Davey PC, Crombe F, Benhabiles N, Holvoet P. Oxidized low-density lipoprotein-induced expression of ABCA1 in blood monocytes precedes coronary atherosclerosis and is associated with plaque complexity in hypercholesterolemic pigs. J Thromb Haemost. 2007;5:2529-36.

Giannarelli C, Wong CK. Crosstalk between inflammatory cells to promote Cardioprotective angiogenesis. J Am Coll Cardiol. 2019;73:3003-5.

Hafiane A, Genest J. ATP binding cassette A1 (ABCA1) mediates microparticle formation during high-density lipoprotein $(\mathrm{HDL})$ biogenesis. Atherosclerosis. 2017;257:90-9.

Han X, Kitamoto S, Lian Q, Boisvert WA. Interleukin-10 facilitates both cholesterol uptake and efflux in macrophages. J Biol Chem. 2009;284:32950-8.

Hecht I, Rong J, Sampaio AL, et al. A novel peptide agonist of formyl-peptide receptor-like 1 (ALX) displays anti-inflammatory and cardioprotective effects. J Pharmacol Exp Ther. 2009;328:426-34.

Hu YW, Ma X, Li XX, et al. Eicosapentaenoic acid reduces ABCA1 serine phosphorylation and impairs ABCA1-dependent cholesterol efflux through cyclic AMP/protein kinase a signaling pathway in THP-1 macrophage-derived foam cells. Atherosclerosis. 2009;204:e35-43.

Jeppesen DK, Fenix AM, Franklin $J$, et al. Reassessment of exosome composition. Cell. 2019;177(428-445):e418.

Kalinec F, Webster P, Maricle A, et al. Glucocorticoid-stimulated, transcriptionindependent release of annexin A1 by cochlear Hensen cells. Br J Pharmacol. 2009;158:1820-34.

Kiani-Esfahani A, Kazemi Sheykhshabani S, Peymani M, Hashemi MS, Ghaedi K, Nasr-Esfahani MH. Overexpression of Annexin A1 suppresses proinflammatory factors in PC12 cells induced by 1-Methyl-4-Phenylpyridinium. Cell J. 2016:18:197-204

Kreutter G, Kassem M, El Habhab A, et al. Endothelial microparticles released by activated protein C protect beta cells through EPCR/PAR1 and annexin A1/ FPR2 pathways in islets. J Cell Mol Med. 2017;21:2759-72.
Krimbou L, Denis M, Haidar B, Carrier M, Marcil M, Genest J Jr. Molecular interactions between apoE and ABCA1: impact on apoE lipidation. J Lipid Res. 2004;45:839-48.

Kuang HJ, Zhao GJ, Chen WJ, et al. Hsp27 promotes ABCA1 expression and cholesterol efflux through the PI3K/PKCzeta/Sp1 pathway in THP-1 macrophages. Eur J Pharmacol. 2017;810:57-62.

Kusters DH, Chatrou ML, Willems BA, et al. Pharmacological treatment with Annexin $A 1$ reduces atherosclerotic plaque burden in LDLR-/- mice on Western type diet. PLoS One. 2015;10:e0130484.

Li L, Xu L, Chen W, et al. Reduced Annexin A1 secretion by ABCA1 causes retinal inflammation and ganglion cell apoptosis in a murine glaucoma model. Front Cell Neurosci. 2018;12:347.

Libby P, Buring JE, Badimon L, et al. Atherosclerosis. Nat Rev Dis Primers. 2019;5: 56.

Locatelli I, Sutti S, Jindal A, et al. Endogenous annexin A1 is a novel protective determinant in nonalcoholic steatohepatitis in mice. Hepatology. 2014;60: 531-44.

Loyer X, Vion AC, Tedgui A, Boulanger CM. Microvesicles as cell-cell messengers in cardiovascular diseases. Circ Res. 2014;114:345-53.

Ma L, Dong F, Denis M, Feng Y, Wang MD, Zha X. Ht31, a protein kinase a anchoring inhibitor, induces robust cholesterol efflux and reverses macrophage foam cell formation through ATP-binding cassette transporter A1. J Biol Chem. 2011;286:3370-8.

Nadkarni S, Cooper D, Brancaleone V, Bena S, Perretti M. Activation of the annexin A1 pathway underlies the protective effects exerted by estrogen in polymorphonuclear leukocytes. Arterioscler Thromb Vasc Biol. 2011;31:2749-59.

Nandi S, Ma L, Denis M, et al. ABCA1-mediated cholesterol efflux generates microparticles in addition to HDL through processes governed by membrane rigidity. J Lipid Res. 2009;50:456-66.

Omer S, Meredith D, Morris JF, Christian HC. Evidence for the role of adenosine $5^{\prime}$-triphosphate-binding cassette (ABC)-A1 in the externalization of annexin 1 from pituitary folliculostellate cells and ABCA1-transfected cell models. Endocrinology. 2006;147:3219-27.

Orekhov AN, Oishi Y, Nikiforov NG, et al. Modified LDL particles activate inflammatory pathways in monocyte-derived macrophages: transcriptome analysis. Curr Pharm Des. 2018:24:3143-51.

Pan B, Kong J, Jin J, et al. A novel anti-inflammatory mechanism of high density lipoprotein through up-regulating annexin A1 in vascular endothelial cells. Biochim Biophys Acta. 2016;1861:501-12.

Parente L, Solito E. Annexin 1: more than an anti-phospholipase protein. Inflamm Res. 2004;53:125-32.

Parisi JDS, Correa MP, Gil CD. Lack of endogenous Annexin A1 increases mast cell activation and exacerbates experimental atopic dermatitis. Cells. 2019;8:51.

Peng $\mathrm{R}$, Ji H, Jin L, et al. Macrophage-based therapies for atherosclerosis management. J Immunol Res. 2020;2020:8131754.

Perretti M, Christian H, Wheller SK, et al. Annexin I is stored within gelatinase granules of human neutrophil and mobilized on the cell surface upon adhesion but not phagocytosis. Cell Biol Int. 2000;24:163-74

Pessolano E, Belvedere R, Bizzarro V, et al. Annexin A1 contained in extracellular vesicles promotes the activation of keratinocytes by Mesoglycan effects: an autocrine loop through FPRs. Cells. 2019;8:753.

Phillips MC. Is ABCA1 a lipid transfer protein? J Lipid Res. 2018;59:749-63.

Poon IK, Lucas CD, Rossi AG, Ravichandran KS. Apoptotic cell clearance: basic biology and therapeutic potential. Nat Rev Immunol. 2014;14:166-80.

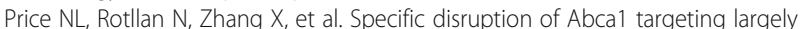
mimics the effects of miR-33 knockout on macrophage cholesterol efflux and atherosclerotic plaque development. Circ Res. 2019;124:874-80.

Purvis GSD, Collino M, Loiola RA, et al. Identification of AnnexinA1 as an endogenous regulator of RhoA, and its role in the pathophysiology and experimental therapy of Type-2 diabetes. Front Immunol. 2019a;10:571.

Purvis GSD, Solito E, Thiemermann C. Annexin-A1: therapeutic potential in microvascular disease. Front Immunol. 2019b;10:938.

Qin C, Yang YH, May L, et al. Cardioprotective potential of annexin-A1 mimetics in myocardial infarction. Pharmacol Ther. 2015;148:47-65.

Raulf N, Lucarelli P, Thavaraj S, et al. Annexin A1 regulates EGFR activity and alters EGFR-containing tumour-derived exosomes in head and neck cancers. Eur J Cancer. 2018;102:52-68

Ray M, Gabunia K, Vrakas CN, et al. Genetic deletion of IL-19 (Interleukin-19) exacerbates Atherogenesis in II19(-/-)xLdlr(-/-) double knockout mice by dysregulation of mRNA stability protein HuR (human antigen R). Arterioscler Thromb Vasc Biol. 2018;38:1297-308. 
Reiss AB, Vernice NA, Siegart NM, De Leon J, Kasselman LJ. Exosomes in cholesterol metabolism and atherosclerosis. Cardiovasc Hematol Disord Drug Targets. 2017;17:185-94.

Rentero C, Blanco-Munoz P, Meneses-Salas E, Grewal T, Enrich C. Annexinscoordinators of cholesterol homeostasis in endocytic pathways. Int J Mol Sci. 2018;19:1444.

Saigusa R, Winkels H, Ley K. T cell subsets and functions in atherosclerosis. Nat Rev Cardiol. 2020

Sanches JM, Branco LM, Duarte GHB, et al. Annexin A1 regulates NLRP3 Inflammasome activation and modifies lipid release profile in isolated peritoneal macrophages. Cells. 2020;9:926.

Seidel S, Neymeyer $H$, Kahl T, et al. Annexin A1 modulates macula densa function by inhibiting cyclooxygenase 2. Am J Physiol Renal Physiol. 2012;303:F84554.

Senchenkova EY, Ansari J, Becker F, et al. Novel role for the AnXA1-Fpr2/ALX signaling Axis as a key regulator of platelet function to promote resolution of inflammation. Circulation. 2019;140:319-35.

Solito E, Christian HC, Festa M, et al. Post-translational modification plays an essential role in the translocation of annexin A1 from the cytoplasm to the cell surface. FASEB J. 2006;20:1498-500

Suades R, Padro T, Crespo J, et al. Liquid biopsy of extracellular microvesicles predicts future major ischemic events in genetically characterized familial hypercholesterolemia patients. Arterioscler Thromb Vasc Biol. 2019:39:117281.

Sugimoto MA, Vago JP, Teixeira MM, Sousa LP. Annexin A1 and the resolution of inflammation: modulation of neutrophil recruitment, apoptosis, and clearance. J Immunol Res. 2016;2016:8239258.

Tang SL, Chen WJ, Yin K, et al. PAPP-A negatively regulates ABCA1, ABCG1 and SR-B1 expression by inhibiting LXRalpha through the IGF-I-mediated signaling pathway. Atherosclerosis. 2012;222:344-54.

Tsai WH, Chien HY, Shih $\mathrm{CH}$, et al. Annexin A1 mediates the anti-inflammatory effects during the granulocytic differentiation process in all-trans retinoic acid-treated acute promyelocytic leukemic cells. J Cell Physiol. 2012;227: 3661-9.

Tsai WH, Lai SL, Li IT, et al. Annexin A1 mediates the anti-adhesive effects of the dexamethasone-treated promyelocytic leukemic cells. J Cell Biochem. 2013; 114:551-7.

Tsuboi T, Lu R, Yonezawa T, et al. Molecular mechanism for nobiletin to enhance ABCA1/G1 expression in mouse macrophages. Atherosclerosis. 2020;297:32-9.

Wang D, Hiebl V, Xu T, et al. Impact of natural products on the cholesterol transporter ABCA1. J Ethnopharmacol. 2020;249:112444.

Wang D, Wang W, Lin W, et al. Apoptotic cell induction of miR-10b in macrophages contributes to advanced atherosclerosis progression in ApoE -/- mice. Cardiovasc Res. 2018b;114:1794-805.

Wang $H$, Yang $Y$, Sun $X$, et al. Sonodynamic therapy-induced foam cells apoptosis activates the phagocytic PPARgamma-LXRalpha-ABCA1/ABCG1 pathway and promotes cholesterol efflux in advanced plaque. Theranostics. 2018c;8:4969-84.

Wang Q, Ren M, Feng F, Chen K, Ju X. Treatment of colon cancer with liver X receptor agonists induces immunogenic cell death. Mol Carcinog. 2018a;57: 903-10.

Wang S, Gulshan K, Brubaker G, Hazen SL, Smith JD. ABCA1 mediates unfolding of apolipoprotein $\mathrm{Al} \mathrm{N}$ terminus on the cell surface before lipidation and release of nascent high-density lipoprotein. Arterioscler Thromb Vasc Biol. 2013:33:1197-205.

Wang Y, Xie Y, Zhang A, Wang M, Fang Z, Zhang J. Exosomes: an emerging factor in atherosclerosis. Biomed Pharmacother. 2019;115:108951.

Wein S, Fauroux M, Laffitte J, et al. Mediation of annexin 1 secretion by a probenecid-sensitive ABC-transporter in rat inflamed mucosa. Biochem Pharmacol. 2004;67:1195-202.

Wu YD, Cui S, Li Q, Zhang RS, Song ZM, Gao YZ, Chen WJ, Xing DG. Recent advances in duplex-specific nuclease-based signal amplification strategies for microRNA detection. Biosens Bioelectron. 2020;165:112449.

Xiao Z, Shen J, Zhang L, Li M, Hu W, Cho C. Therapeutic targeting of noncoding RNAs in hepatocellular carcinoma: recent progress and future prospects. Oncol Lett. 2018;15:3395-402

Yin K, Chen WJ, Zhou ZG, et al. Apolipoprotein A-I inhibits CD40 proinflammatory signaling via ATP-binding cassette transporter A1-mediated modulation of lipid raft in macrophages. J Atheroscler Thromb. 2012;19:823-36.
Yu XH, Zhang DW, Zheng XL, Tang CK. Cholesterol transport system: an integrated cholesterol transport model involved in atherosclerosis. Prog Lipid Res. 2019;73:65-91.

Zhang M, Wu JF, Chen WJ, et al. MicroRNA-27a/b regulates cellular cholesterol efflux, influx and esterification/hydrolysis in THP-1 macrophages. Atherosclerosis. 2014;234:54-64.

Zhou L, Choi HY, Li WP, Xu F, Herz J. LRP1 controls CPLA2 phosphorylation, ABCA1 expression and cellular cholesterol export. PLoS One. 2009;4:e6853.

\section{Publisher's Note}

Springer Nature remains neutral with regard to jurisdictional claims in published maps and institutional affiliations.
Ready to submit your research? Choose BMC and benefit from:

- fast, convenient online submission

- thorough peer review by experienced researchers in your field

- rapid publication on acceptance

- support for research data, including large and complex data types

- gold Open Access which fosters wider collaboration and increased citations

- maximum visibility for your research: over $100 \mathrm{M}$ website views per year

At BMC, research is always in progress.

Learn more biomedcentral.com/submissions 